\title{
The Effective Thermal Conductivity of Crud and Heat Transfer from Crud-coated PWR Fuel
}

\author{
N. Cinosi, I. Haq, M. Bluck, S.P. Walker \\ Mechanical Engineering Department \\ Imperial College, London \\ n.cinosi@imperial.ac.uk
}

\begin{abstract}
:
Water-filled crud on the surface of PWR fuel could offer resistance to the flow of heat, which might be expected to cause higher clad temperatures, and probably more fuel failures, than are actually observed. However, there is some evidence from post-irradiation inspection that the crud is penetrated by pores large enough to permit vapour formation, and it is believed these provide a mechanism for 'wick boiling' to occur, improving heat transfer. This phenomenon is investigated using a two-dimensional coupled multi-physics model, accounting for the flow of water, heat and dissolved species within the crud. The fuel thermal performance is characterized in terms of an effective crud thermal conductivity derived from the use of this model, and the non-linear dependence this effective conductivity has on parameters such as crud thickness and pore density is determined.
\end{abstract}

\section{INTRODUCTION}

Most PWR fuel becomes coated with various deposits, collectively termed 'crud' [1]. It takes a variety of physical and chemical forms, that change over the life of the fuel, but largely comprises corrosion products from primary circuit components. It is generally believed to play a role in fuel failure; empirically, fuel cladding corrosion failure tends to correlate well to the presence and thickness of crud. The mechanism for this is uncertain, but is believed to be some combination of (i) the crud may raise the local clad temperature, and (ii) aggressive dissolved species are concentrated in the crud [2-5]. Studies of crud feature, for example, in the "Zero fuel failure by 2010 " INPO initiative.

Simple models of the thermal resistance of wet crud predict temperatures that would be expected to cause more fuel failures than are observed, and it is believed that a 'wick boiling' mechanism is at work [6]. In this paper we use a newly developed, two-dimensional coupled wick-boiling heat and mass transfer model to investigate the thermal hydraulic effect of the crud. We use its predictions to identify conditions when wick boiling is expected to dominate, and associate with this mechanism an 'effective' thermal conductivity, to facilitate comparison with reactor-scale experimental measurements.

In Section 2 we consider the thermal hydraulic effects of crud, and the cladding temperatures to be expected in the absence of wick boiling. In Section 3 we summarise the coupled heat and mass transfer wick boiling model, and in Section 4 we apply this model to identify the conditions under which wick boiling is to be expected, and the effective thermal conductivity' it corresponds to.

\section{THE THERMAL HYDRAULIC EFFECTS OF CRUD}

\subsection{Crud formation}

There is a wide variety of physical and chemical forms and species that collectively are termed 'crud', and this diversity naturally make analysis and quantification of its effects uncertain. There is an extensive literature on the subject; a selection that gives the most useful background for present purposes is cited here. In particular, a very helpful description of the various (and changing) forms of crud is provided by [1], as part of a discussion of the difficulties inherent in the identification and characterization of crud formation over the $\sim 50,000$ fuel rods of a PWR. 


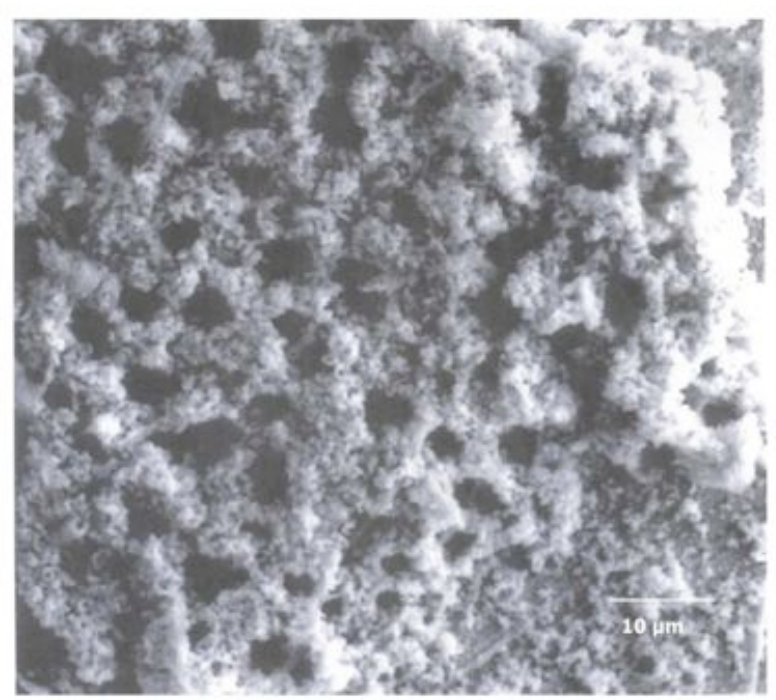

Figure 1

Top view of PWR crud. (Extent of photograph about 60 microns). Note the presence of 'chimneys' [7].
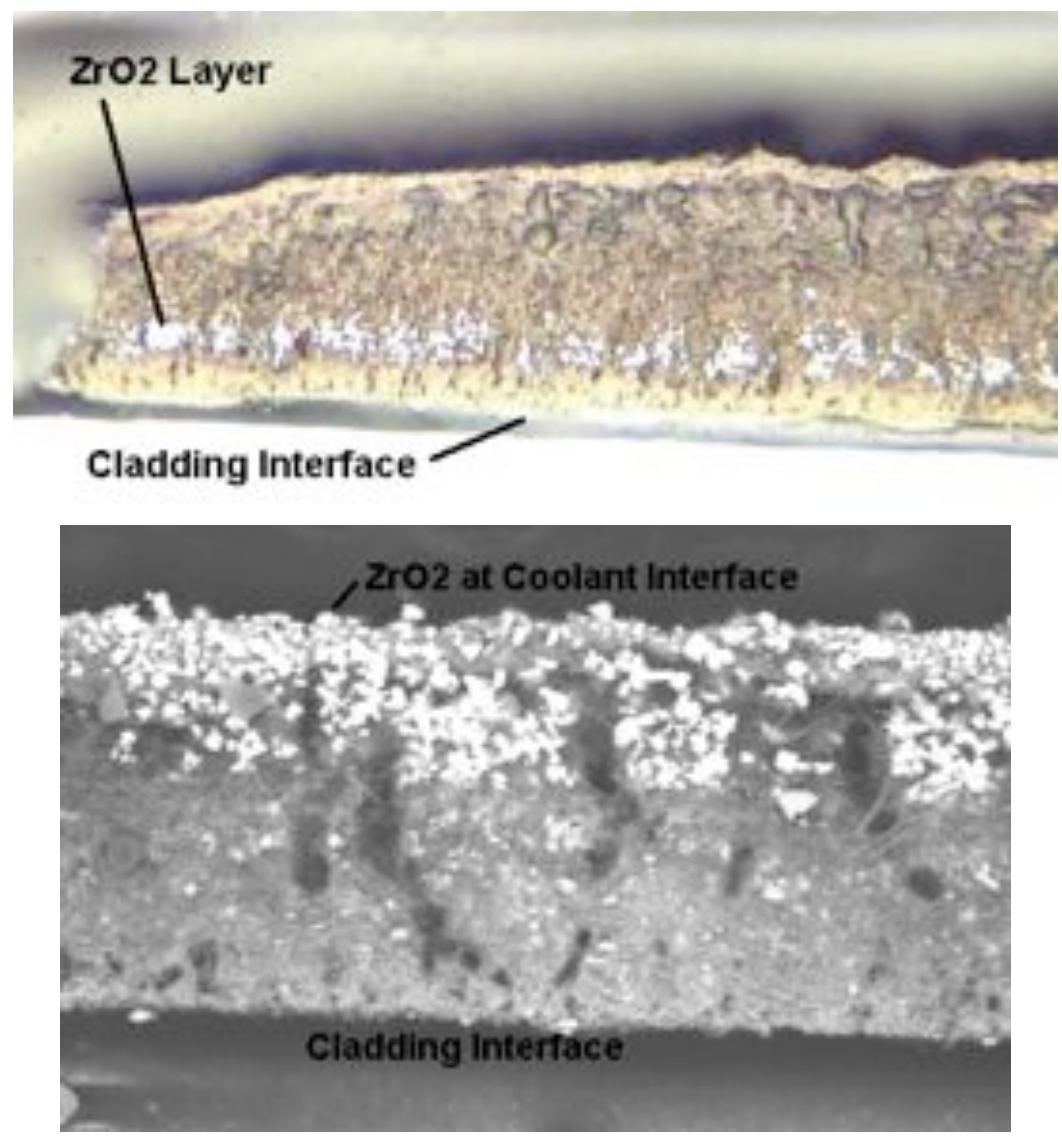

Figure 2

Sections through crud. Note the sectioned 'chimneys'. Photographs reproduced from Byers[1]

\subsection{Heat transfer from crud-coated fuel}

The surface roughness of the fuel rod, and the associated coolant pressure drop and fuelcoolant heat transfer coefficient will all be influenced by the presence of the crud. We will not 
address this aspect further here, but rather will focus on the transmission of heat through the crud layer.

The conductivity of the fluid-filled crud will be estimated, in terms of the thermal conductivities of the two phases, and the void fraction $\varepsilon$ in the crud, from the Maxwell formula [8]:

$$
k_{C R U D}=k_{F L U I D}\left\{\frac{1-\left(1-a \frac{k_{\text {SOLID }}}{k_{F L U I D}}\right)(1-\varepsilon)}{1+(a-1)(1-\varepsilon)}\right\}
$$

where

$$
a=\left(\frac{3 k_{\text {FLUID }}}{2 k_{\text {FLUID }}+k_{\text {SOLID }}}\right)
$$

In Figure 3 is indicated the consequent dependence of crud conductivity on porosity. For relevant porosities, the conductivity is dominated by that of the fluid. Vapour-filled crud is seen, naturally, to have a conductivity less than half that of water-filled crud. 


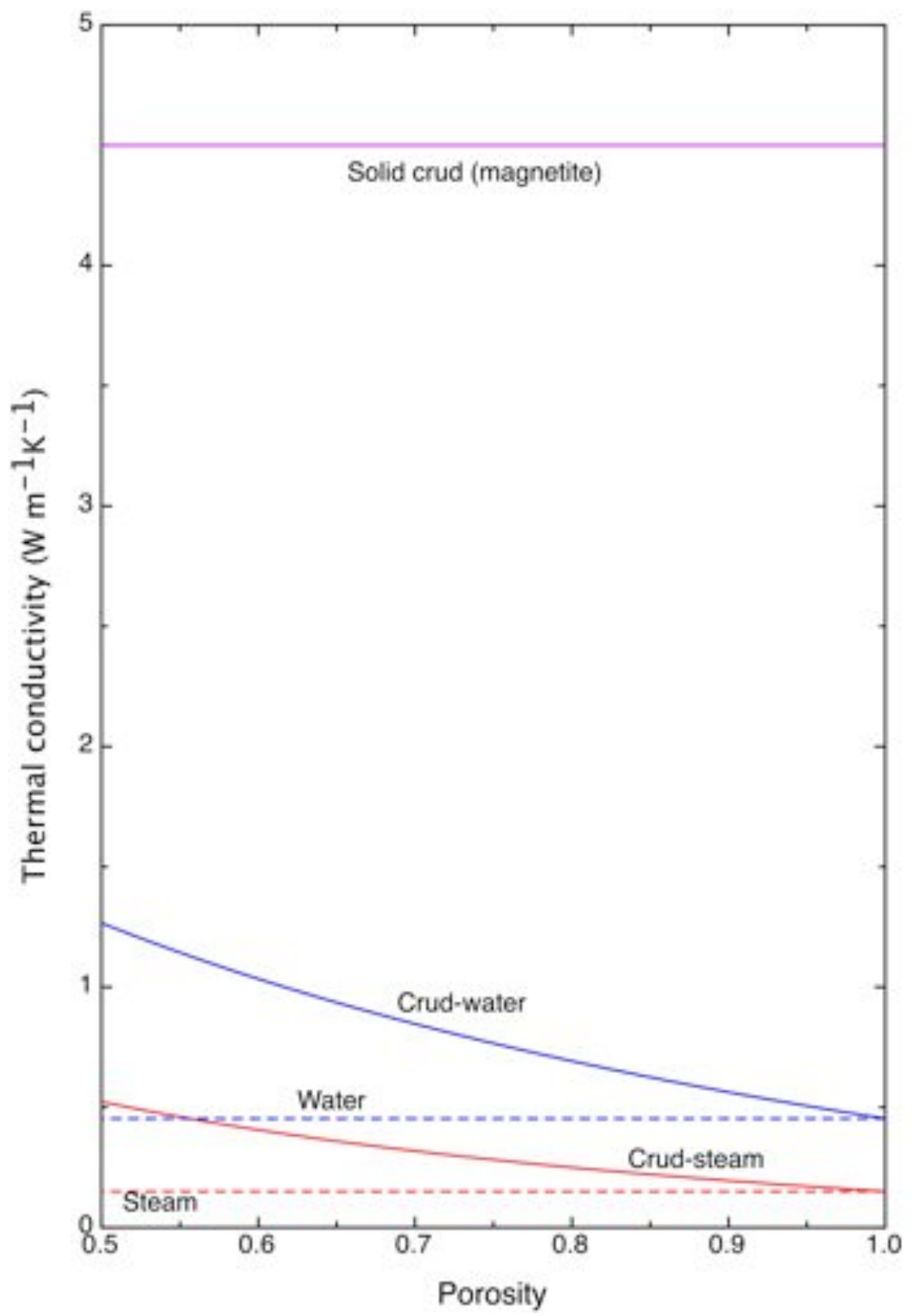

Figure 3

Crud thermal conductivity, as a function of porosity, with the porosity occupied by water and water vapour. Also indicated are the conductivity of solid crud, taken as magnetite, and of water and steam.

One mechanism for the transmission of heat to the coolant is by conduction through the crud. The crud in effect adds an additional 'thermal resistance', analogous, for example, to the thermal resistance presented by the pellet-clad gap.

We will here identify the modification to the fuel's radial temperature profile caused by the crud coating. Using in (1) and (2) the parameters from Table 1 yield a water-filled crud conductivity of $0.692 \mathrm{Wm}^{-1} \mathrm{~K}^{-1}$. In Figure 4 we show the temperature profiles in the presence of crud layers of 32 and 64 microns in depth. The clad surface temperature is increased considerably, by about $3 \mathrm{~K}$ per micron of crud.

For the circumstances considered here, the bulk saturation temperature, indicated as a horizontal line on the figure, is reached about 10 microns into the crud. Even though the small pore sizes will inhibit boiling until some temperature higher than this, there will be a layer of vapour-filled crud inboard of this for cases with crud significantly more than 10 microns thick. With a thermal conductivity about $1 / 3$ of water-filled crud, this will cause clad temperatures to be higher still. 

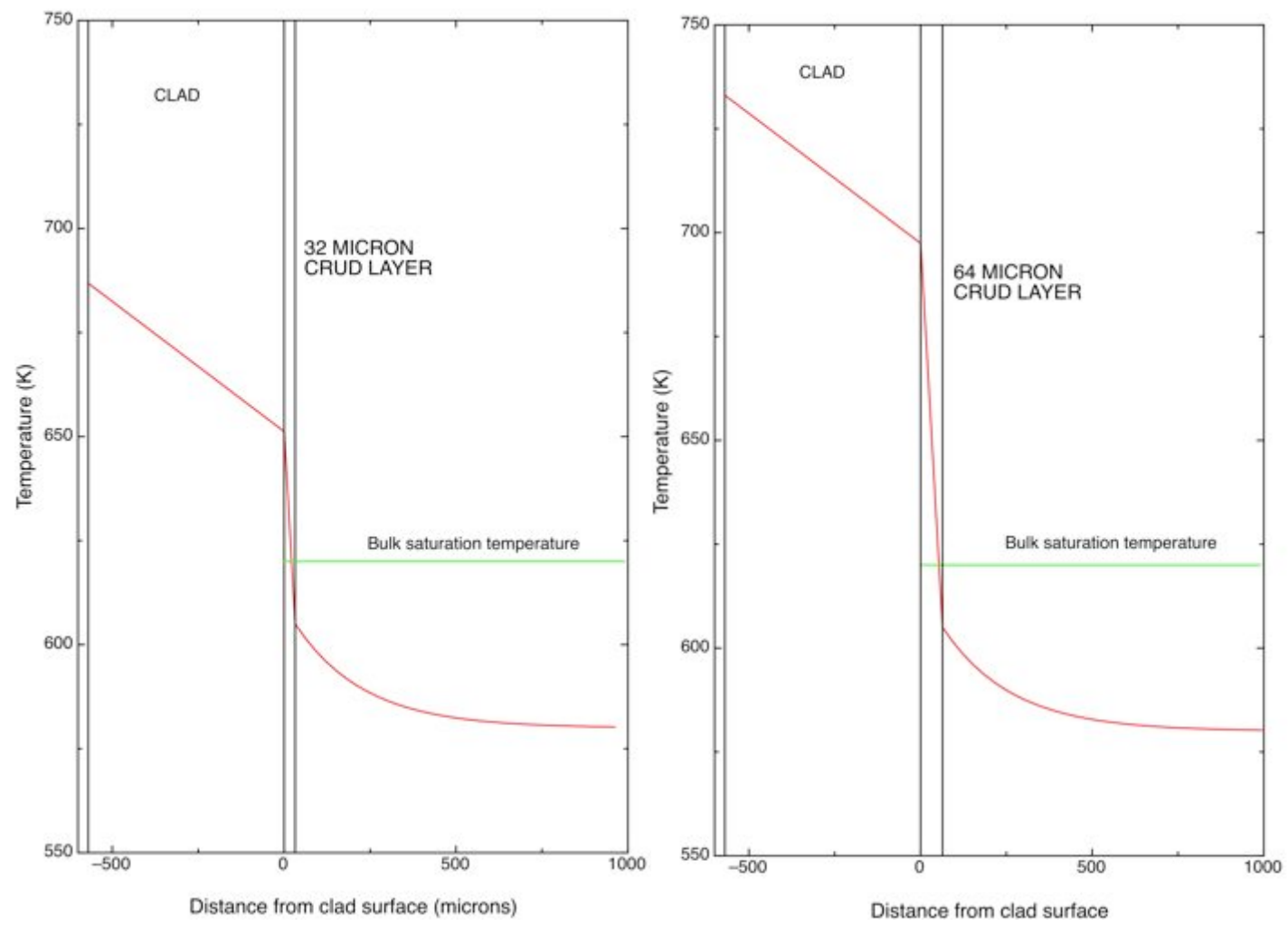

Figure 4

Radial temperature profile with a 30 micron and 60 micron crud layers (no wick boiling)

\subsection{Wick boiling}

The high temperatures that the above analysis indicates would be expected to cause more problems with fuel failure than are actually observed. One possible explanation for this stems from the structure of the crud. Rather than a homogeneous porous solid, post-irradiation examination indicates that the crud contains relatively coarse interconnected passageways. This provides locations where vapourisation can occur, with the vapour then escaping and the water being replenished by diffusive flow. This mechanism was proposed originally by Macbeth [6,9].

The mouths of these pores are visible in Figure 1, and their paths are visible in the sections of Figure 2.

The wick boiling mechanism implies that a complex set of coupled phenomena govern behaviour, including conduction, porous medium flow, and migration within that flow of various dissolved species. More recent work [4] by Henshaw and colleagues has investigated, in particular, the complex chemical interactions associated with this.

Our primary interest here is in the way in which the removal of heat from the crud is facilitated by this, and in how the effective thermal conductivity of the crud is thereby changed. This mechanism provides an explanation as to why the high temperatures suggested above are not expected. A detailed, coupled wick boiling model will be summarized in the following section.

\section{SUMMARY OF THE COUPLED HEAT \& MASS TRANSFER MODEL}

\subsection{Introduction}

We will here give a short summary of the coupled heat and mass transfer model of the processes believed to be occurring in the crud; a fuller description has just been produced [10].

As is plain in Figure 4, water temperatures within the crud would be well in excess of the boiling temperature if it were a homogeneous, water-saturated solid. As noted above, in 
practice large pores ('chimneys') are commonly present, and evaporation thus takes place into these.

An idealized sketch of this is shown in Figure 5. At the face of the chimney water is evaporated, and the steam so produced escapes into the sub-cooled coolant, where it is condensed. Water to replace that evaporated is drawn through the porous crud to the chimney face.

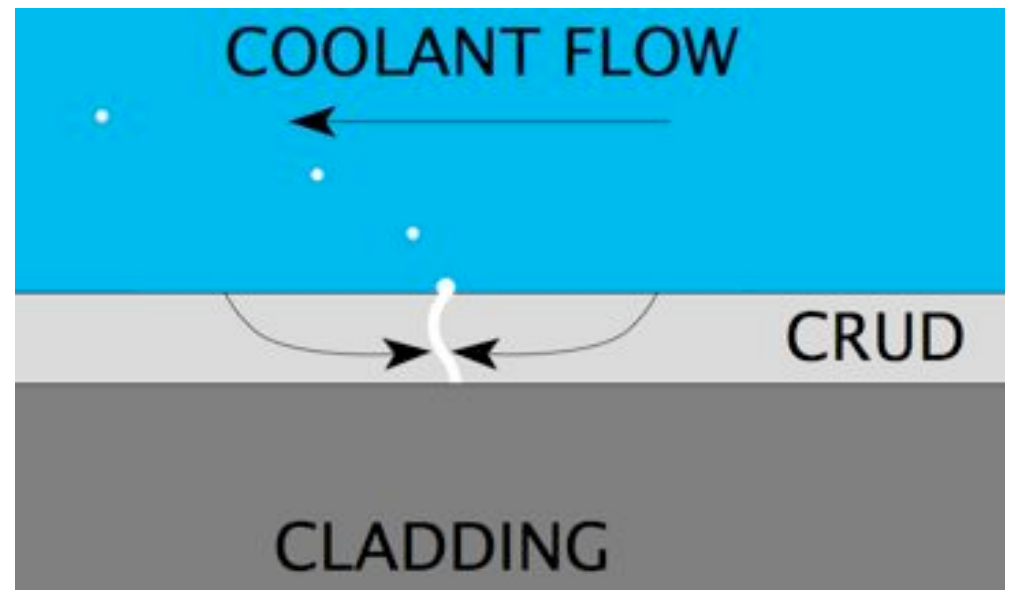

Figure 5

Schematic of the wick boiling process

\subsection{Three coupled processes}

There are three coupled processes in operation here:

Heat conduction: The rate of evaporation of water at the chimney face depends on the temperature distribution on the wet crud, with heat transported by conduction.

The flow of water: The flow of water through the crud is determined by this; water must flow through the crud to replace that evaporated.

Species concentration: This flow will bring with it whatever species are dissolved in the coolant; either deliberately (boron compounds, for example) or as a result of corrosion. Little of the dissolved material will evaporate at the chimney face, and a high concentration will build up there. It will be limited by the diffusion of the dissolved material, back down the concentration gradient this generates.

These three processes are all coupled. The concentrations developed will affect the saturation temperature at the chimney face. This temperature boundary condition influences the distribution of the conduction heat flux, which in turn influences the flow pattern. This flow pattern is responsible for the species concentrations developed, closing the loop of mutual coupled dependence.

\subsection{The model developed}

An idealised two-dimensional coupled model of this has been implemented. An attempt to depict the disposition of crud and chimney, more or less to scale, is given in Figure 6, where in addition the two-dimensional $(r-z)$ solution domain adopted is indicated. 


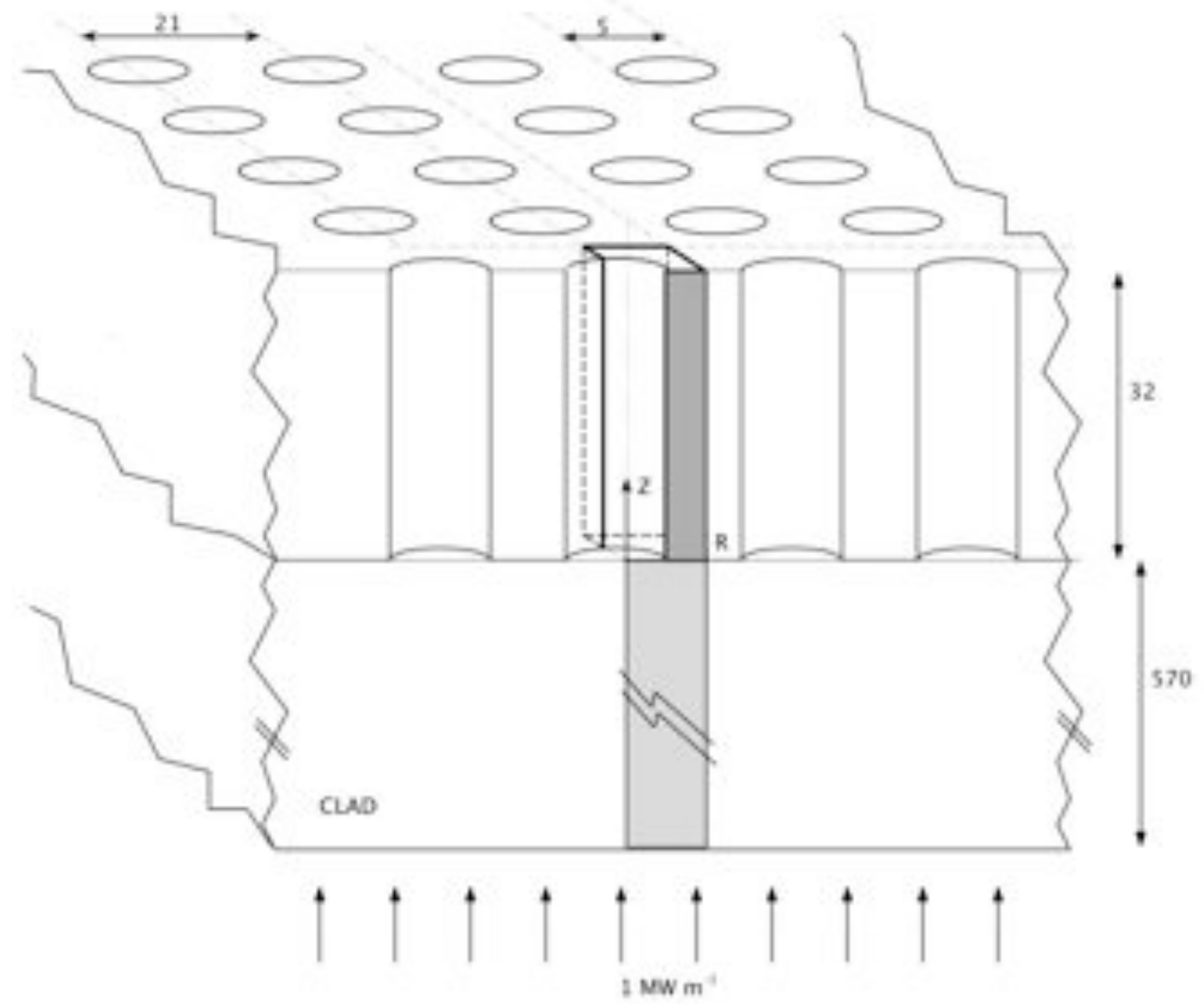

Figure 6

Clad, crud and chimneys, and the axi-symmetric solution domain adopted

The solution domains (indicated in grey), governing equations and boundary conditions for each of the three governing equations are shown in Figure 7.
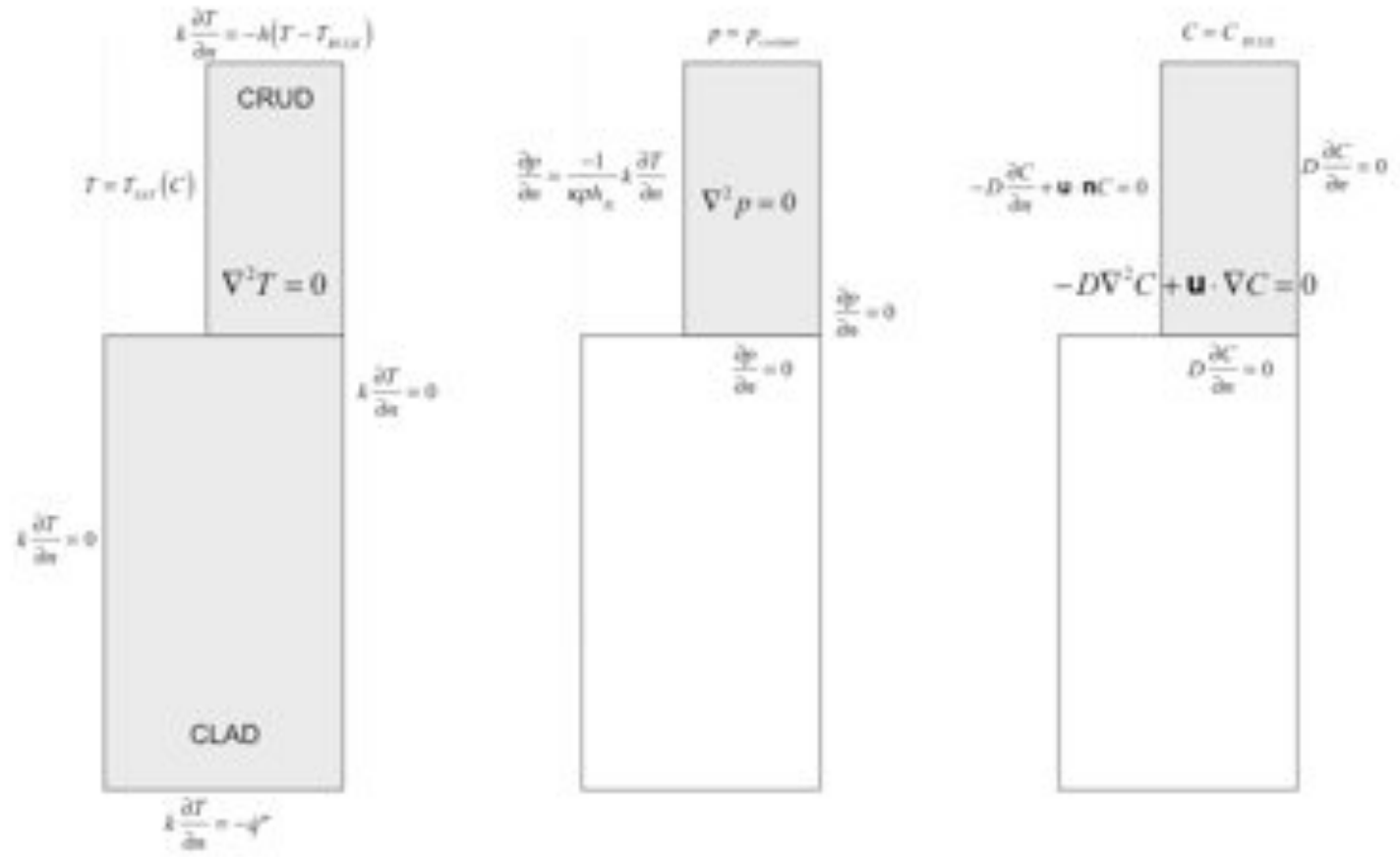
Figure 7

Solution domain, governing equations and boundary conditions of the coupled heat and mass transfer model

Note that the solution domain for the heat conduction analysis extends to include the clad. It turns out that omission of the clad alters predictions of solute concentrations by a factor of about two.

\section{WICK-BOILING BEHAVIOUR}

\subsection{A note on the calculations}

As will already be apparent, the composition and morphology of crud is very varied. It is also uncertain. Inter alia, the solubility of much of the material from which it is formed varies with temperature and pressure, which means that the crud present under reactor conditions may well have characteristics (such as pore sizes) significantly different from those of the crud on which measurements are eventually made in a laboratory. The thermal conductivity of the solid phase is uncertain, reflecting its uncertain composition, even if it were present in bulk as a homogeneous material. This is further compounded by its chaotic morphology.

An inevitable consequence of all this is that numerical values of model predictions will have large uncertainties. A realistic aim of such analysis, perhaps, will be to attempt to identify the main processes and work, their relative importance, and their dependence on the major parameters.

The numerical values of the parameters employed in the present illustrative calculations are listed in Table 1.

\begin{tabular}{|l|l|l|}
\hline Crud thickness & $\mu m$ & 32 \\
\hline Chimney centres & $\mu m$ & 21 \\
\hline Chimney diameter & $\mu m$ & 5 \\
\hline Crud porosity & $\%$ & 80 \\
\hline Crud solid conductivity & $\mathrm{Wm} \cdot \mathrm{K}^{-1}$ & 4.5 \\
\hline Tortuosity of porosity & & 0.4 \\
\hline Coolant boron concentration & $\mathrm{ppm}\left(\mathrm{gm}^{-3}\right)$ & 4637 \\
\hline Coolant liquid conductivity & $\mathrm{Wm} \cdot \mathrm{K}^{-1}$ & 0.453 \\
\hline Coolant vapour conductivity & $\mathrm{Wm} \cdot \mathrm{K}^{-1}$ & 0.15 \\
\hline Bulk coolant temperature & $\mathrm{K}$ & 590 \\
\hline Heat flux & $\mathrm{MWm}^{-2}$ & 1.0 \\
\hline System pressure & $\mathrm{MPa}^{-15}$ & 15.5 \\
\hline Coolant heat transfer coefficient & $\mathrm{W} \mathrm{m} \cdot \mathrm{K}^{-1}$ & $1.2 \mathrm{E} 04$ \\
\hline
\end{tabular}

Table 1

Values of parameters employed in the analyses presented here (unless stated otherwise for individual studies)

\subsection{Temperature and heat flux distributions predicted by the coupled two dimensional wick boiling model}

We show in Figure 8 the predictions of the two-dimensional coupled model for the case characterized by these parameter values.

For completeness, we have shown results for the predicted concentrations of dissolved boron, showing it to reach a multiple of $\sim 25$ over the concentration in the bulk coolant. This does increase the saturation temperature, and thereby modifies the predicted effective clad 
conductivity, but this is perhaps less important than its effect on local chemistry. This is beyond the scope of this present paper, and we will not refer further to the species concentrations.
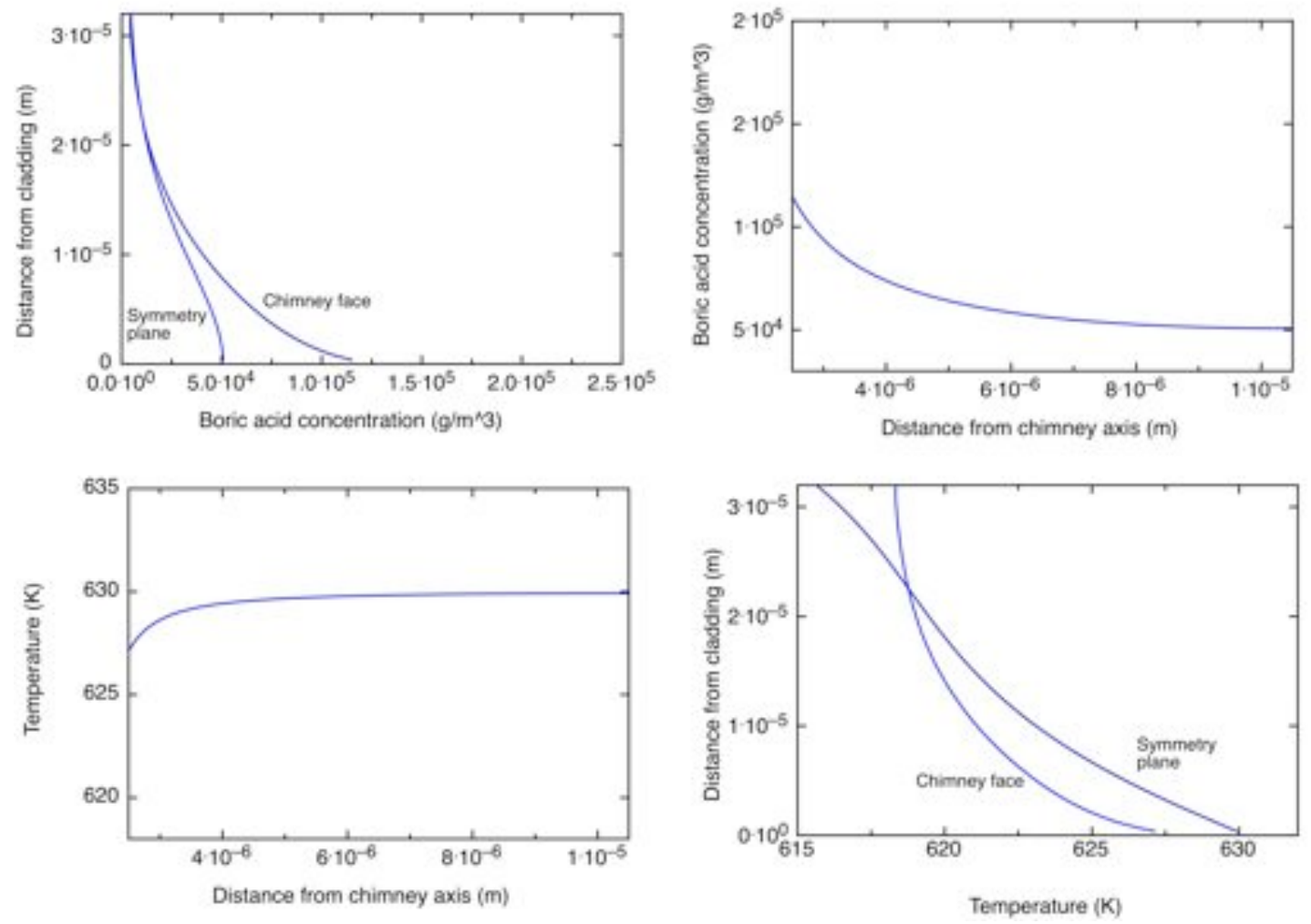

Figure 8

Temperature and species concentration variation within the crud

A contour plot of temperatures, and a vector plot of heat flow, is shown in Figure 9, where we indicate the temperature distribution and heat flows within, and to and from, the crud.
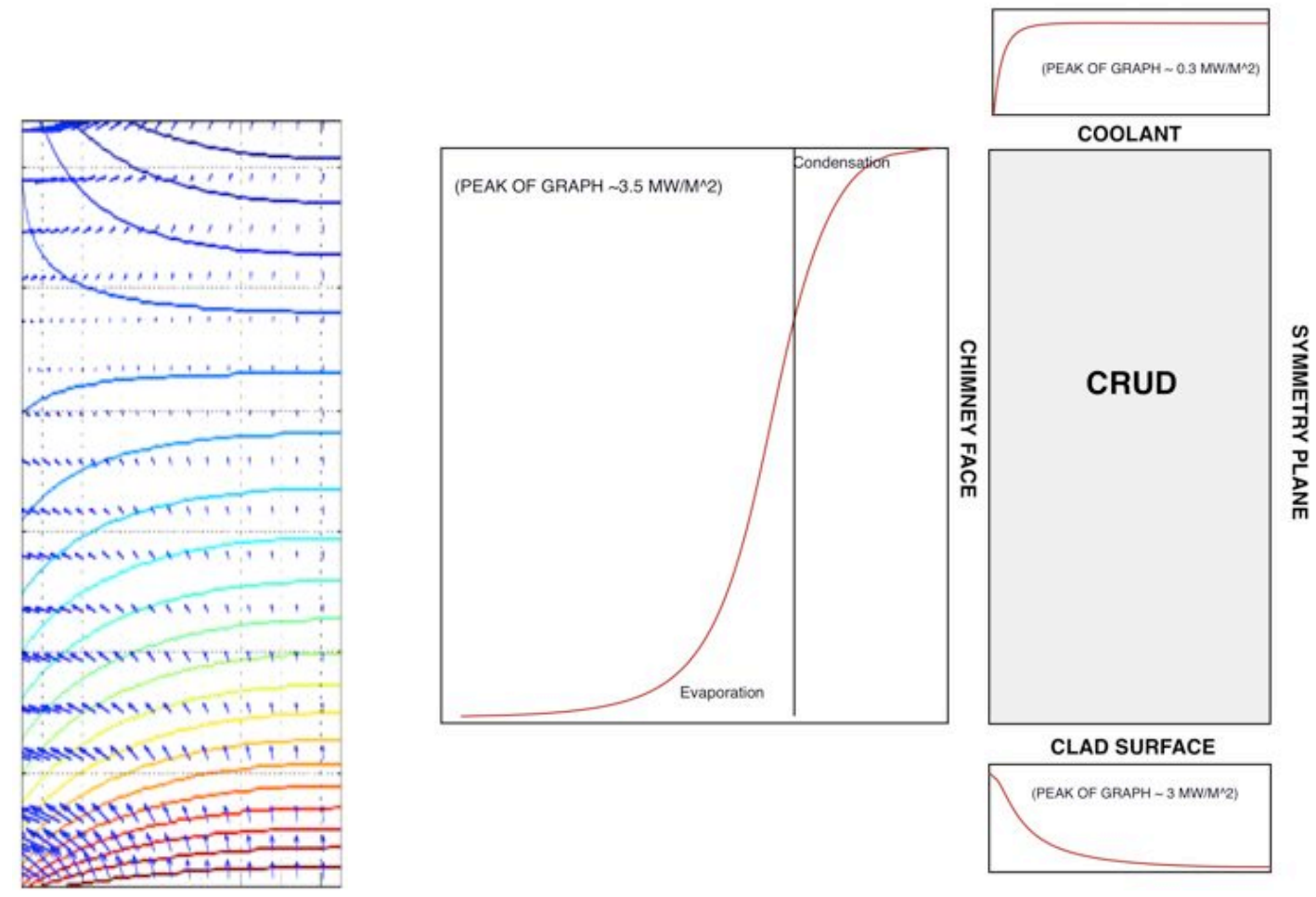
Figure 9

Left: Contour plot of temperatures, and a plot of heat flow vectors, in the crud. Left side chimney face, right side symmetry plane. Right: A depiction of the surface heat fluxes into the chimney, and into the bulk coolant from the wetted crud surface.

Plainly, the mechanism that is overwhelmingly responsible for the removal of heat from the clad to the coolant is radial conduction towards the chimneys, with evaporative cooling at the chimney face. (Interestingly, the model here predicts a little condensation towards the upper part of the chimney. The magnitude of this is sensitive to the modeling of, for example, the coolant heat transfer coefficient, and it is only present for a limited range of parameter values.) For the conditions employed here, about $70 \%$ of the heat is removed via net evaporation in the chimney. Associated with this, it is seen that the heat flux from the clad surface is very localized around the periphery of the chimney.

We will now seek to identify the circumstances under which this mechanism, of heat removal primarily by wick boiling, is expected to be dominant. We will then attempt to characterise the behaviour, expressing it in terms of an 'effective thermal conductivity' for the crud. One reason for the significance of this characterization is that it is really only this that can be measured in reactor-relevant large-scale experiments, and an understanding of its appropriateness and helpfulness is necessary.

\subsection{When is wick boiling likely?}

Plainly, if the chimneys are separated by many crud thicknesses, we would not expect their influence to extend over the whole fuel surface; the thermal behaviour might be expected to tend towards that depicted in Figure 4. We will now perform analyses with a range of separations to determine the chimney density required to cause wick boiling to be the dominant mechanism for heat removal.

From Figure 9 we see that there is only a fairly small temperature variation along the clad crud interface, and along the crud - coolant interface. We will express and analyse results in terms of (weighted) averages of each of these temperatures, allowing, for example, an 'equivalent' crud thermal conductance to be defined.

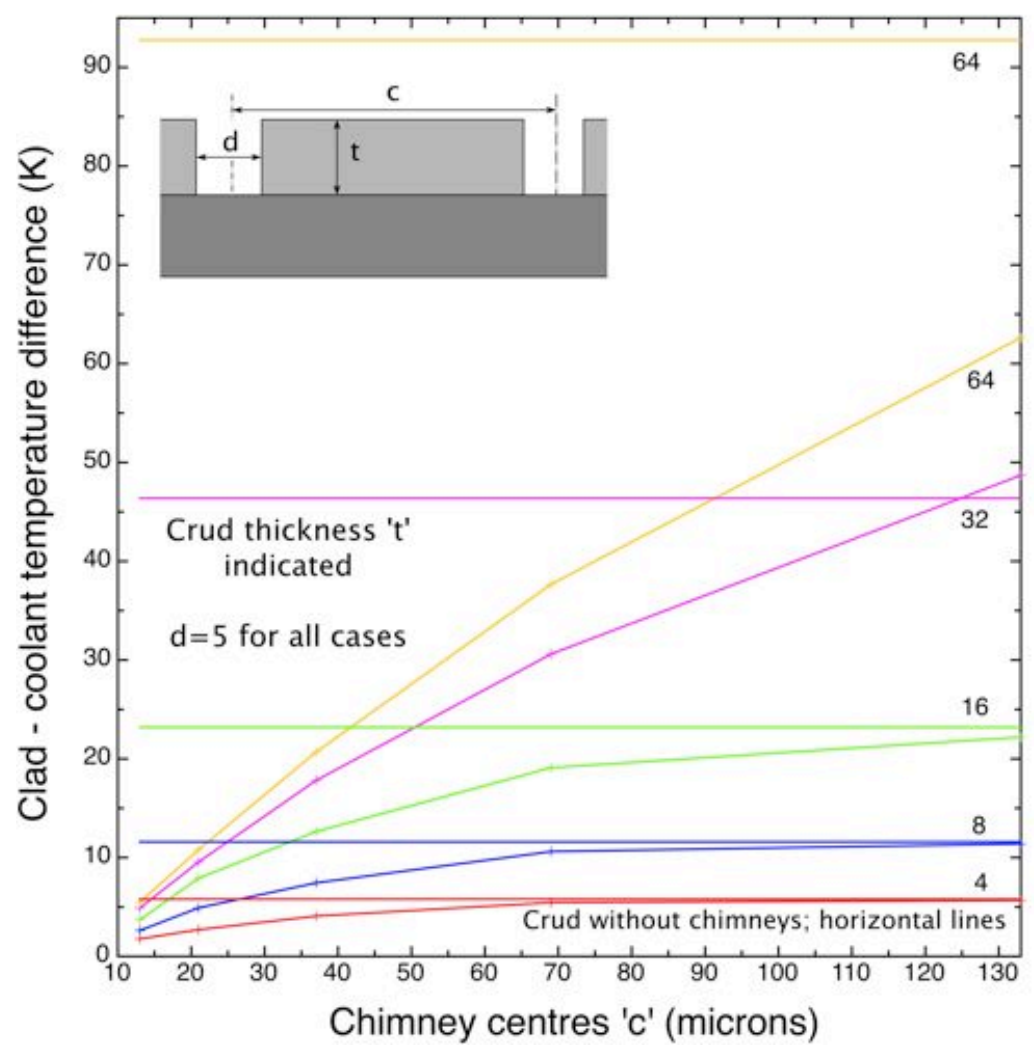

Figure 10

Clad to coolant mean temperature difference versus chimney centres, for a range of clad 
thicknesses. Also shown are the temperature differences that would be present in the case of no chimneys

In Figure 10 we show the model's prediction of the temperature difference, averaged over the surface, between the metal-crud and crud-water interfaces. Also shown are the corresponding temperature differences for the case of no chimneys. As the chimney separation increases, the temperature difference approaches the 'normal' values, as predicted by the wet crud model discussed above. The chimney spacing at which this occurs is greater for thick crud, as would be expected. For smaller chimney separations, the temperature rise through the crud is seen to be very much smaller than the simple wet crud model would predict.

The same data is presented in a different form in Figure 11. Considering for example the wellseparated chimneys of the orange line, with chimney centres of 133 microns, it is not until a crud thickness of $\sim 30$ microns that any perturbation from the 'normal' wet crud is observed. For more closely spaced chimneys, the temperature rise through the crud is much reduced by the chimneys. It is notable that the temperature of the cladding is predicted to be only weakly influenced by the thickness of the crud. This is a distinct departure from the 'wet crud' prediction.

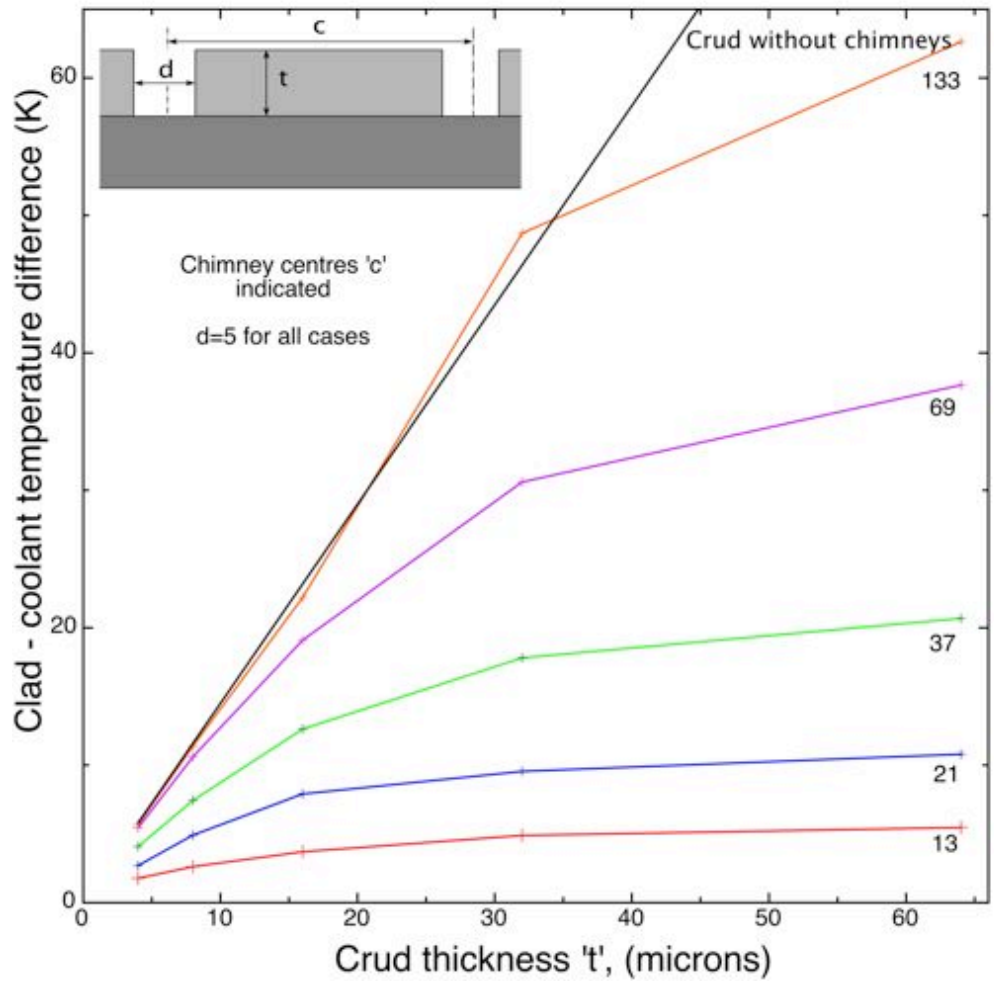

Figure 11

Clad to coolant mean temperature difference versus crud thickness, for a range of chimney centres. Also shown is the variation of temperature difference that would be present in the case of no chimneys

\subsection{Effective crud conductivity}

In reactor-scale measurements of the thermal effects of crud the most natural quantity to try to measure is the temperature difference across the clad, the crud and the water-side boundary layer. (Indeed, the local bulk coolant temperature may, in addition, itself be inferred from energy conservation arguments.) From this a crud thermal conductivity can be estimated. This is one of the uses of the 'WALT' rig, for example [11].

We can use the results of the model to estimate equivalent crud thermal conductivity.

In Figure 12 is shown the dependence of effective thermal conductivity on crud thickness and chimney spacing. It is plain that crud thermal conductivity is very much not a 'material property', but varies strongly -by some orders of magnitude- according to the local geometry. 
Observed crud chimneys generally have separations of a few microns to ten or so microns, which indicates that this mechanism will generally be applicable.
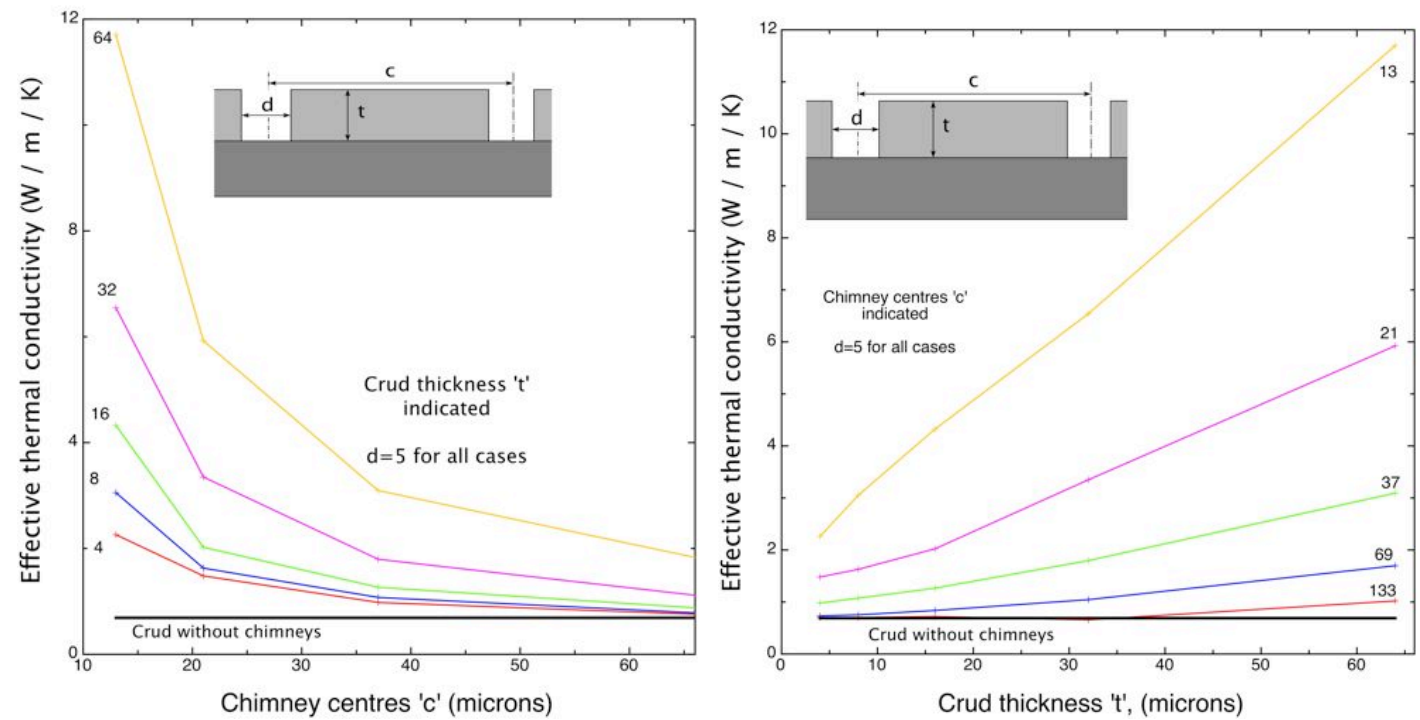

Figure 12

Crud effective thermal conductivity as a function of chimney separation and crud thickness. The conductivity of chimney-free wet crud is indicated also.

It is convenient to combine the results, to give an approximate, but fairly robust, indication of the dependence of effective conductivity on the main parameters that affect it; the crud thickness, and the chimney separation. This is shown in Figure 13. It is clear that the wick boiling mechanism has the ability to reduce clad temperatures significantly, over a wide parameter space.

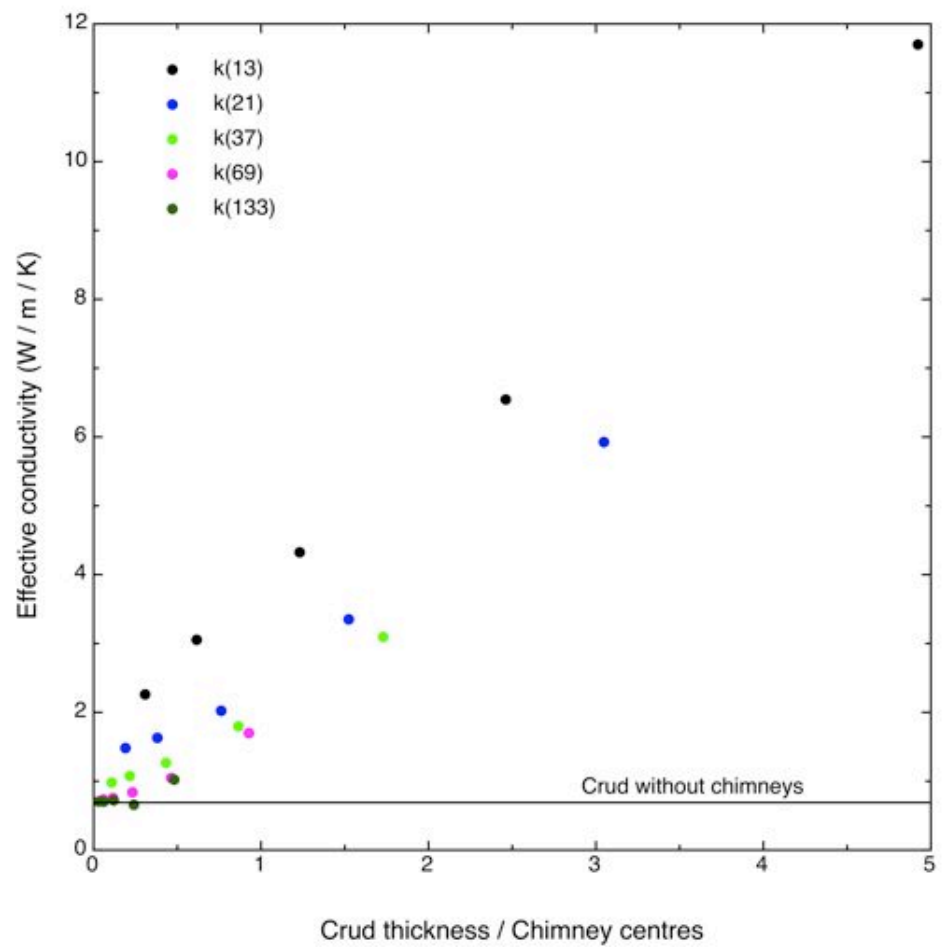

Figure 13

The dependence of effective conductivity on crud thickness and chimney separation, obtained by aggregating the results of the previous analyses. The numbers in the legend indicate to which values of crud centre each data point corresponds 


\section{CONCLUSIONS}

It has been shown that the usual 'porous medium' model of thermal conductivity predicts values that would cause very high clad temperatures, even if vapour-film generation does not occur.

The role of wick boiling in crud 'chimneys' has been investigated using a coupled twodimensional model. Whilst a major thrust of this model is the investigation of the movement and concentration of the dissolved species from the coolant into the crud, where they can be concentrated by factors of 25 and above, attention here has focused on the associated thermal consequences.

The dramatic effect wick boiling has on the thermal performance has been quantified. The behaviour of the crud coating has been characterized, pragmatically, in terms of an 'effective thermal conductivity', whilst noting that the heat transfer processes at work are overwhelmingly NOT thermal conduction. The dependence of this effective conductivity on the main parameter that affects it, namely the chimney separation, has been quantified. The predicted temperature difference through a wide range of cruds (of crud thicknesses, and chimney separations), is actually less than, or similar to, the reduction in the water-side boundary layer temperature drop, suggesting that if anything the presence of crud might be expected to reduce clad temperatures.

The results and methods presented will contribute to interpretation of reactor-scale measurements of crud thermal conductivity.

\section{REFERENCES}

[1] W. A. Byers, "Pressurized water reactor core crud mapping," in 18th international conference on nuclear engineering (ICONE 18), Xian China, 2010.

[2] P. Cohen, "Heat and mass transfer for boiling in porous deposits with chimneys," AICHE Symposium series, vol. 70, pp. 71-80, 1974.

[3] J. Deshon, "Pwr axial offset anamoly (aoa), guidelines, revision 1," EPRI, Palo Alto, CA 1008102, 2004.

[4] J. Henshaw, et al., "A model of chemistry and thermal hydraulics in PWR fuel crud deposits," Journal of Nuclear Materials, vol. 353, pp. 1-11, 2006.

[5] C. Pan, et al., "Concentration levels of solutes in porous deposits with chimneys under wick boiling conditions," Nuclear Engineering and Design, vol. 99, pp. 317-327, 1987.

[6] R. V. Macbeth, "Boiling on surfaces overlayed with a porous deposit: Heat transfer rates obtained by capillary action," Atomic Energy Establishment Winfrith AEEW-R711, 1971.

[7] J. Mahlon, "The Simulation and study of conditions leading to axial offset anomaly in pressurized water reactors," M.Sc Thesis M.Sc Thesis, Georgia Institute of Technology, USA, 2004.

[8] C. Pan and B. G. Jones, "Wick boiling performance in porous deposits with chimneys," in AICHE/ANS National heat transfer conference symposium on multiphase and heat transfer, Denver, 1985.

[9] R. V. Macbeth and et al., "An investigation into the effect of crud deposits on surface temperature, dry out and pressure drop, with forced convection boiling of water at 69 bar in an annular test section," Atomic Energy Establishment Winfrith AEEW-R 705, 1971.

[10] I.Haq, et al., "Modelling heat transfer and dissolved species concentrations within PWR crud," in Nuclear Engineering and Design, ed, 2010.

[11] G. Wang, et al., "Methods to reduce CIPS/ CILC risk for the zero fuel failure by 2010 initiative," in 18th international conference on nuclear engineering (ICONE 18), Xian China, 2010. 